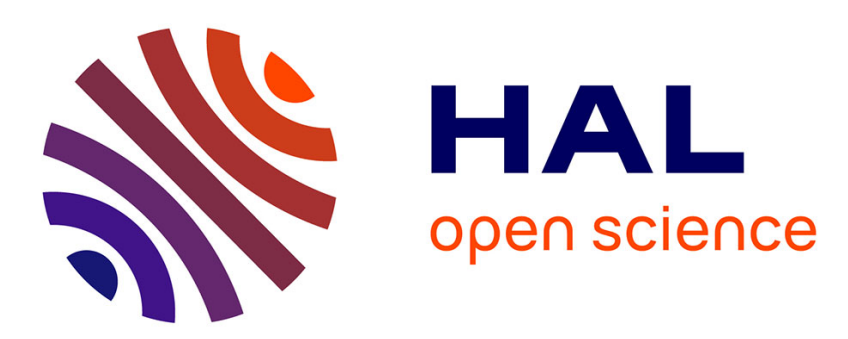

\title{
OSCILLATOR STRENGTH MEASUREMENTS BY THE EMISSION METHOD : NI AND CI LINES IN THE 1200-2000 Å SPECTRAL RANGE
}

C. Goldbach, G. Nollez

\section{- To cite this version:}

C. Goldbach, G. Nollez. OSCILLATOR STRENGTH MEASUREMENTS BY THE EMISSION METHOD : NI AND CI LINES IN THE 1200-2000 A SPECTRAL RANGE. Journal de Physique IV Proceedings, 1991, 01 (C1), pp.C1-83-C1-87. 10.1051/jp4:1991111 • jpa-00249749

HAL Id: jpa-00249749

https://hal.science/jpa-00249749

Submitted on 1 Jan 1991

HAL is a multi-disciplinary open access archive for the deposit and dissemination of scientific research documents, whether they are published or not. The documents may come from teaching and research institutions in France or abroad, or from public or private research centers.
L'archive ouverte pluridisciplinaire HAL, est destinée au dépôt et à la diffusion de documents scientifiques de niveau recherche, publiés ou non, émanant des établissements d'enseignement et de recherche français ou étrangers, des laboratoires publics ou privés. 


\title{
OSCILLATOR STRENGTH MEASUREMENTS BY THE EMISSION METHOD : NI AND CI LINES IN THE 1200-2000 \& SPECTRAL RANGE
}

\author{
C. GOLDBACH and G. NOLLEZ \\ CNRS, Institut d'Astrophysique, 98 bis Boulevard Arago, F-75014 Paris, \\ France
}

\begin{abstract}
Résumé- Les principes et la réalisation d'une expérience de mesure de forces d'oscillateur dans l'ultraviolet lointain par une méthode d'émission sont brièvement présentés. Les forces d'oscillateur de sept raies fortes et de onze raies faibles de NI ainsi que de vingt-trois raies fortes et de vingt raies faibles de CI ont été mesurées dans le domaine spectral 1200-2000 $\AA$. L'échelle absolue établie à partir de ces mesures permet une comparaison avec les résultats théoriques récents.

Abstract- The principles and the realisation of an experiment devoted to oscillator strength measurements in the vacuum-ultraviolet by an emission method are briefly presented. The oscillator strengths of seven strong NI lines, eleven weak NI lines, twenty-three strong CI lines and twenty weak CI lines have been measured in the 1200- $2000 \AA$ spectral range. The absolute scale set up from the measurements is compared with recent theoretical results.
\end{abstract}

This paper presents the principles, the realisation and the results of an experiment devoted to oscillator strength measurements in the vacuum-ultraviolet (VUV).

At the time of the conception of this experiment in the early 80's, many atomic data were missing to analyse the high-resolution spectra obtained in the VUV from the Copernicus and IUE satellites; even for elements as abundant in the universe as $\mathrm{C}, \mathrm{N}$ and $\mathrm{O}$ there was a lack of accurate $g f$-values. By the same time the experimental techniques largely improved and it became possible to extend to the VUV the classical emission method of oscillator strength measurement without any dramatic loss of precision. The technical improvements relate to crucial experimental points: realisation of high-resolution VUV spectrometers, of VUV radiation standards, computer control in data acquisition and analysis. Also the accuracy reached now on the lifetime data allows the normalisation to an absolute scale of the measured relative $f$-values.

Our program is to measure relative oscillator strengths in the VUV, mainly for neutral and singly ionized carbon, nitrogen and oxygen; this paper resumes the results obtained for the neutral carbon and nitrogen lines in the $1200-2000 \AA$ spectral region.

\section{The experiment}

We use a wall-stabilised arc as the emission source; it burns in argon under LTE conditions $\left(T_{e}=13000 \mathrm{~K} ; n_{e}=1.110^{17} \mathrm{~cm}^{-3}\right)$ and it remains stable when a small amount of gaseous compound like $\mathrm{CO}_{2}$ or $\mathrm{N}_{2}$ is introduced to study neutral and singly ionised species. End-on observations of the axial temperature-homogeneous plasma region are performed through the anodes using a $2.5 \mathrm{~m}$ normal incidence monochromator equipped with a holographic concave grating (measured resolution around $0.06 \AA$ with $10 \mu \mathrm{m}$ entrance and exit slits). A solar blind photomultiplier (CsTe photocathode) serves as detector. The evacuated VUV spectroscopic system is isolated from the arc chamber by a $\mathrm{MgF}_{2} \mathrm{window}$. A measurement and control processor is interfaced between the experiment and a minicomputer.

The absorption of the strong lines in the cool gas layer between the plasma and the $\mathrm{MgF}_{2}$ window is the main experimental difficulty met in this work. We use high-purity argon flowing through the arc channel from the anodes to the cathodes at a high flow rate while a small amount of $\mathrm{CO}_{2}$ or $\mathrm{N}_{2}$ mixed in 
Ar is introduced at the middle of the column at very low flow rates. Under these conditions we succeed to prevent any diffusion of the investigated elements in the cooler zone.

\section{The method}

We set up for the measurements an advanced version of the emission method avoiding the difficulties associated with an absolute number density determination in a multi-component plasma. The method is based on relative oscillator strength measurements and a reliable and precise value of the oscillator strength of the line standing for the reference is required. Fortunately, as can be seen on table 1, the lifetimes of the upper term of the NI multiplet at $1200 \AA$ and of the CI multiplet at $1561 \AA$ are known at the present time with a good accuracy: these lifetimes have been measured through different techniques and the results converge in a very satisfying manner. The line oscillator strengths are deduced directly (branching fraction $=1$ for these multiplets) assuming LS-coupling.

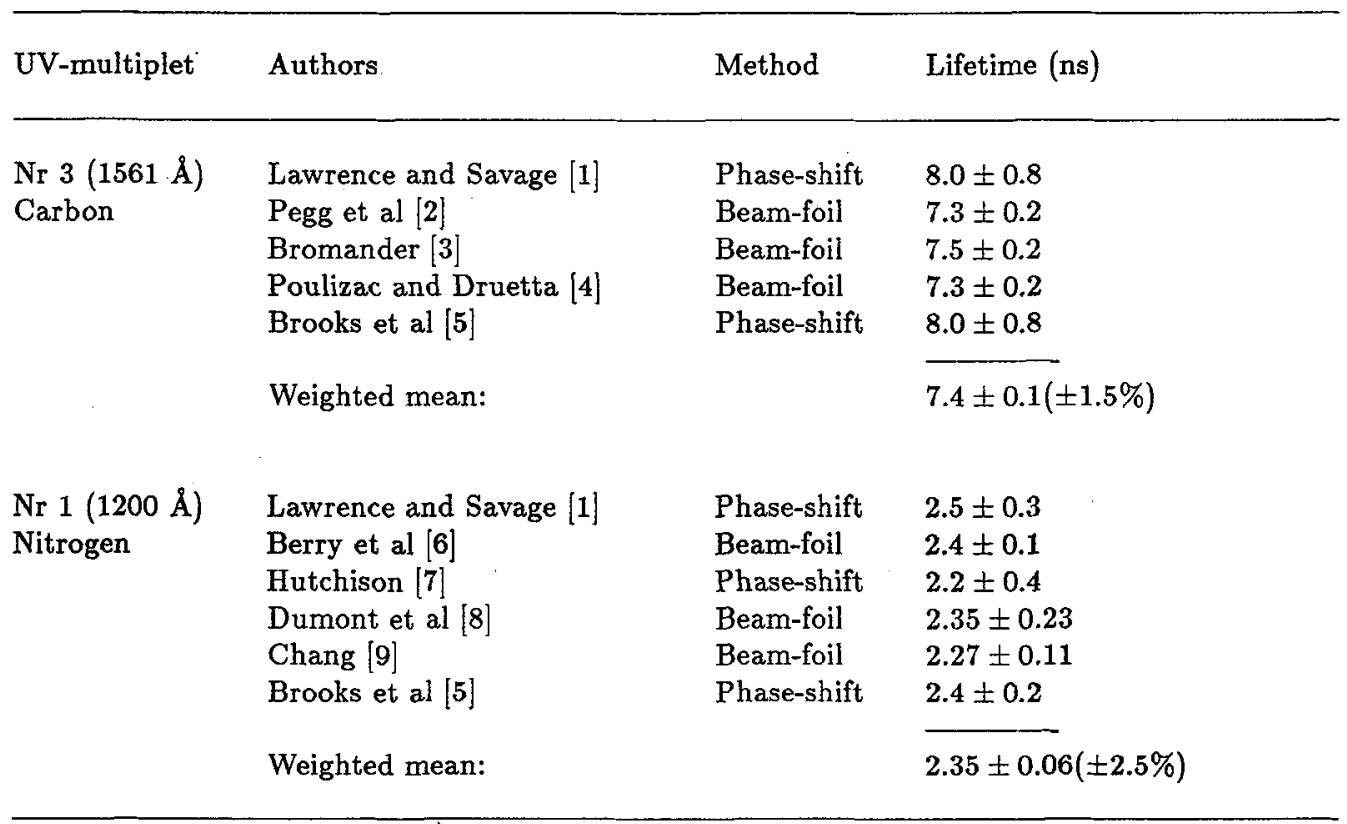

Table 1. Lifetime measurements of the upper terms of two selected CI and NI multiplets.

Depending on the strength of the investigated line the measurement of the oscillator strength is performed following two different ways:

In the case of the strong lines, the line optical depth is not negligible. To take full account of this effect, the quantity we choose to determine is the optical depth profile of the line $\tau_{\lambda}$ (see [10] and [11]). The relative oscillator strength $f_{X} / f_{R}$ of a line $\mathrm{X}$ ( $f_{R}$ being the oscillator strength of the reference line) is deduced, the plasma temperature being independently measured, from the ratio of the integrated optical depths of the two lines:

$$
\frac{\int_{L} \tau_{\lambda}^{X} d \lambda}{\int_{L} \tau_{\lambda}^{R} d \lambda}=\frac{\lambda_{X}^{2}}{\lambda_{R}^{2}} \frac{(g f)_{X}}{(g f)_{R}} \exp \left(\frac{E_{R}^{l o}-E_{X}^{l o}}{k T}\right) .
$$

$\tau_{\lambda}$ is determined from the end-on measured spectral radiance $I_{\lambda}$ through

$$
\tau_{\lambda}=-\ln \left(1-\frac{I_{\lambda}}{B_{\lambda}}\right)
$$


where $B_{\lambda}$ is the Planck function at the plasma temperature. For the strong lines, the blackbody saturation level $B_{\lambda}$ may be measured at the top of the line by increasing the $\mathrm{CO}_{2}$ or $\mathrm{N}_{2}$ concentration [10] .

The weak lines are not blackbody saturable. Moreover we have checked their optical thinness under the experimental conditions and we measure the spectral radiance profile $I_{\lambda}$ of the line. The $f$-value is now deduced from the ratio

$$
\frac{\int_{L} I_{\lambda}^{X} d \lambda}{\int_{L} \tau_{\lambda}^{R} d \lambda}=\frac{2 h c^{2}}{\lambda_{X}^{3} \lambda_{R}^{2}} \frac{(g f)_{X}}{(g f)_{R}} \exp \left(-\frac{E_{X}^{u p}-E_{R}^{l o}}{k T}\right),
$$

(see [12] and [13]). The radiometric calibration of the spectroscopic system is performed using the continuum radiation of the argon plasma itself as a radiance transfer standard [12].

Many of the investigated transitions appear as multiplets of overlapping and blended lines. An accurate determination of the integrated optical depth or spectral radiance of the individual lines (or groups of completely blended lines) is then performed by least- square fitting of digital data. As Gaussian (Doppler, instrumental) and Lorentzian (Stark) line-broadening processes are all of the same order of magnitude, each line is represented by a Voigt function (four free parameters: Gauss and Lorentz widths, position of the line peak, integrated optical depth or spectral radiance). The analytical function representing the multiplet is obtained by addition of a constant continuum to the sum of the individual Voigt components. The integrated optical depth or spectral radiance of each line is obtained as the parameter final value after convergence of the fitting procedure.

\section{The results}

Systematic errors are mainly related to the uncertainties on the reference $f$-value and on the temperature (which enters the Boltzmann factor), to the residual absorption in the boundary layer as well as to the uncertainties relative to the two different radiometric calibration procedures. The final uncertainty, which includes the statistical error, is between 10 and $15 \%$ for the lines of the strong multiplets and at the $20 \%$ level for the weaker lines.
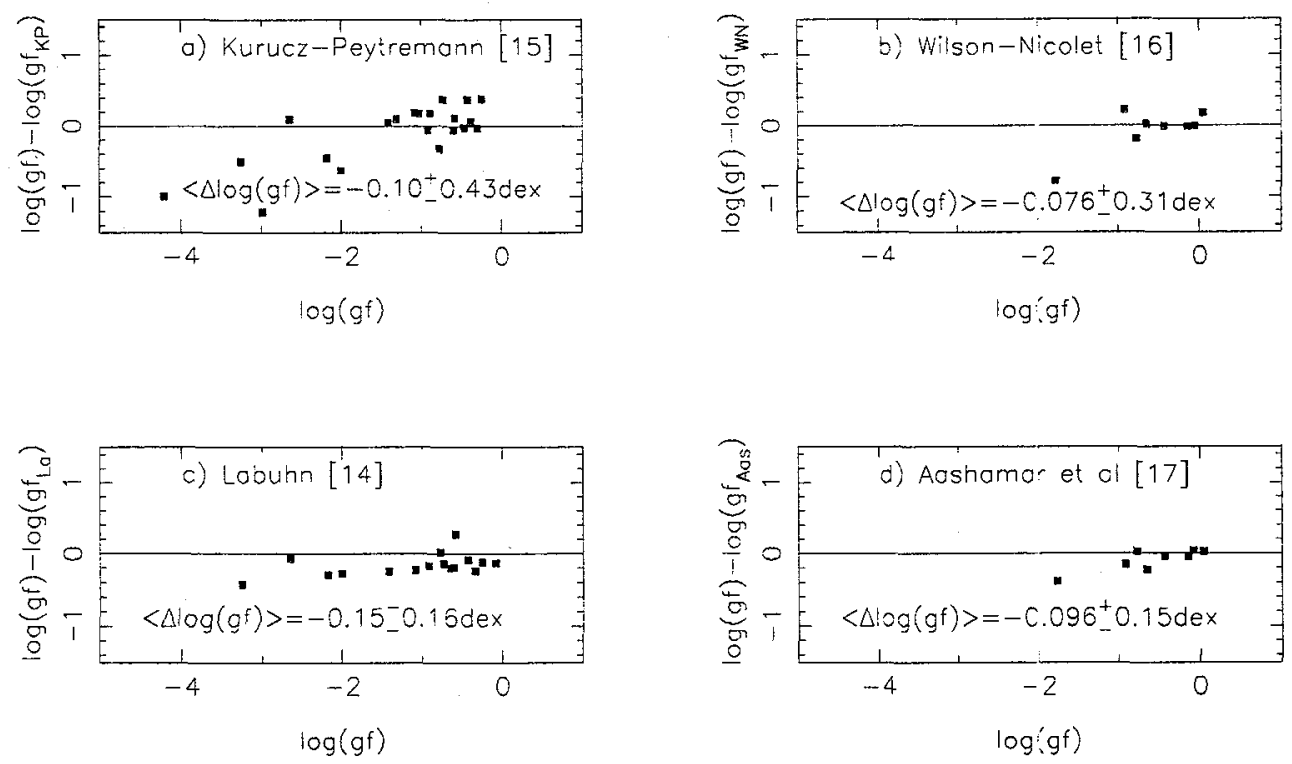

Fig.1: Comparison of our measured NI $g f$-values with other theoretical or experimental data sets.

Fig. 1 summarizes the results obtained for strong and for weak lines of neutral nitrogen in terms of a logarithmic comparison with previous data. The early experimental absolute scale established by Labuhn 
[14] in 1965 appears as overestimated by a mean factor 1.4; as at the time of this experiment no lifetime was available, Labuhn had to perform, in order to get absolute $f$-values, a number density determination in his multi-component plasma (demixing effect). The discrepancies between the two experimental scales are not surprising. The comparison with the semi-empirical results of Kurucz and Peytremann [15], which are widely used by astronomers, shows a large dispersion and also a line strength dependent discrepancy. The Hartree-Fock calculations of Wilson and Nicolet [16] yield multiplet values which exhibit quite a large dispersion. As suggested during the workshop by Dr Grevesse and by Dr Wiese, we present also a comparison with the results of the multiconfiguration optimized potential model by Aashamar et al. [17]: a line-strength (or excitation energy) discrepancy is clearly apparent.
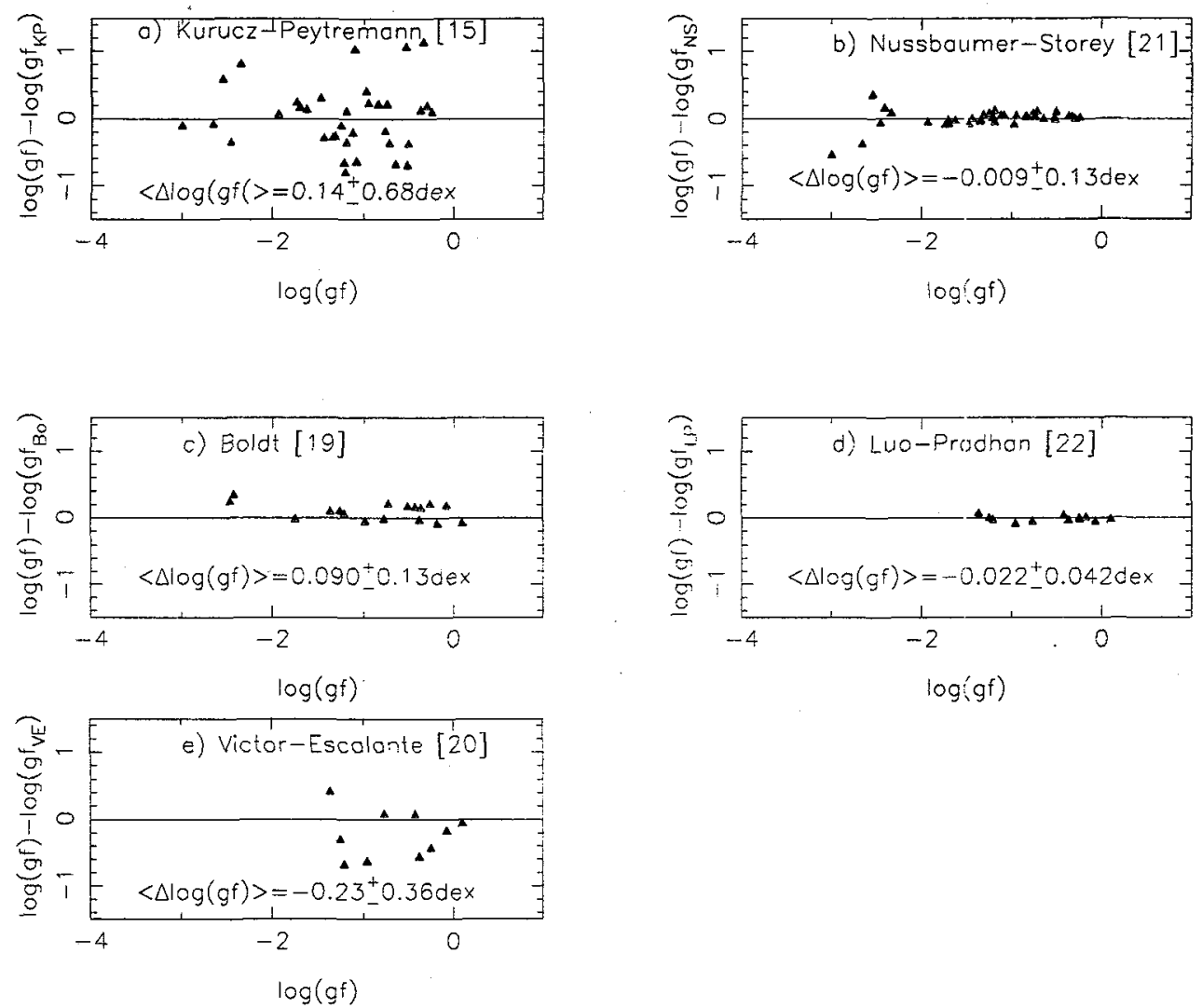

Fig.2: Comparison of our measured CI $g f$-values with other theoretical or experimental data sets.

For the resonance multiplet at $1200 \AA$, configuration interaction calculations by Hibbert et al [18] give results in very good agreement with the mean experimental lifetime we adopted as reference (the other transitions investigated in [18] lie at wavelengths lower than $1200 \AA$ not yet attainable to our measurements).

It should also be mentioned that Opacity results for NI, presented during the workshop by Dr Lennon, appear in good agreement with our experimental scale.

In the case of neutral carbon (Fig.2), the early experiment by Boldt [19], which presents the same sources of errors as the NI experiment of Labuhn, yields an absolute scale 20\% lower than ours. The discrepancies with Kurucz an Peytremann [15] appear again as very large and erratic. Similarly recent calculations by Victor and Escalante [20], using an open-shell semi-empirical model potential method, give results which strongly differ with ours. 
In contrast the agreement between our experimental absolute scale and the multiconfiguration calculations in intermediate coupling of Nussbaumer and Storey [21] is excellent except for the weakest intercombination lines: the mean deviation between the two scales is -0.009 dex $(-2.1 \%)$ or, if the five weakest lines are excluded from the comparison, $0.0049 \mathrm{dex}(1.1 \%)$. Our analysis [13] of the possible experimental sources of error concludes that no specific experimental error may explain the discrepancies for the weakest lines. Lastly, calculations performed in the frame of the Opacity Project by Luo and Pradhan [22] yield multiplet values which agree also very well with our scale: mean deviation between the two scales $-0.022 \operatorname{dex}(-5.1 \%)$.

This very good agreement in the case of CI between our experimental scale, based on measured relative f-values and on measured lifetime data and the theoretical scales established by Nussbaumer and Storey and by Luo and Pradhan is worth emphasizing.

\section{References}

/1/ Lawrence, G.M. and Savage, B.D., Phys. Rev. 141 (1966) 67.

/2/ Pegg, D.J., Chupp, E.L. and Dotchin, L.W., Nucl. Instr. and Meth. 90 (1970) 71.

/3/ Bromander, J., Physica Scripta 4 (1971) 61.

/4/ Poulizac, M.-C., Druetta, M. and Ceyzeriat, P., J. Quant. Spectrosc. Radiat. Transfer 11 (1971) 1087.

/5/ Brooks, N.H., Rohrlich, D. and Smith, W.H., Astrophys. J. 214 (1977) 328.

/6/ Berry, H.G., Bickel, W.S., Bashkin, S., Desesquelles, J. and Schectman, R.M., J. Opt. Soc. Am. 61 (1971) 947.

/7/ Hutchison, R.B., J. Quant. Spectrosc. Radiat. Transfer 11 (1971) 81.

18/ Dumont, P.D., Biemont, E. and Grevesse N., J. Quant. Spectrose. Radiat. Transfer 14 (1974) 1127.

/9/ Chang, M.W., Astrophys. J. 211 (1977) 300.

/10/ Goldbach, C., Martin, M., Nollez, G., Plomdeur, P., Zimmermann, J.P. and Babic̀, D., Astron. Astrophys. 161 (1986) 47.

/11/ Goldbach, C. and Nollez, G., Astron. Astrophys. 181 (1987) 203.

/12/ Goldbach, C. and Nollez, G., Astron. Astrophys. 201 (1988) 189.

/13/ Goldbach, C., Martin, M. and Nollez, G., Astron. Astrophys. 221 (1989) 155.

/14/ Labuhn, F., Z. Naturforsch. 20a (1965) 998.

/15/ Kurucz, R.L. and Peytremann, E., A Table of Semi-empirical gf-Values, Cambridge (Mass.); Smithsonian Institution Astrophysical Observatory (1975).

/16/ Wilson, K.H. and Nicolet, W.E., J. Quant. Spectrosc. Radiat. Transfer 7 (1967) 891.

/17/ Aashamar, K., Luke, T.M. and Talman, J.D., Physica Scripta 27 (1983) 267.

/18/ Hibbert, A., Dufton, P.L. and Keenan, F.P., Monthly Notices Roy. Astron. Soc. 213 (1985) 721.

/19/ Boldt, G., Z. Naturforsch. 18a (1963) 1107.

/20/ Victor, G.A. and Escalante, V., Atomic Data and Nuclear Data Tables 40 (1988) 203.

/21/ Nussbaumer, H. and Storey, P.J., Astron. Astrophys. 140 (1984) 383.

/22/ Luo, D. and Pradhan, A.K., J. Phys. B: At. Mol. Phys. 22 (1989) 3377. 